\title{
Pre-processing Technique using Colour-based Feature Method to Detect Categories of Leaves Disease
}

\author{
Siti Haslinda bt Miasin \\ Faculty of Computer Science and \\ Information Technology \\ Universiti Malaysia Sarawak \\ Kota Samarahan, Malaysia \\ 63798@siswa.unimas.my
}

\author{
Phei Chin LIM* \\ Faculty of Computer Science and \\ Information Technology \\ Universiti Malaysia Sarawak \\ Kota Samarahan, Malaysia \\ pclim@unimas.my
}

\author{
Jacey-Lynn Minoi* \\ Faculty of Computer Science and \\ Information Technology \\ Universiti Malaysia Sarawak \\ Kota Samarahan, Malaysia \\ jacey@unimas.my
}

\begin{abstract}
Oil palm leaves diseases is caused by various plant pathogens and micronutrient deficiency, and genetic disorders. This problem, if not identified and treated quickly could lead to losses in yield and profitability. The disease on leaves is currently being identified through the different colours, shapes, and forms. Other signs of an infected plant can be seen based on the discolouration on the leaves. In this paper, we present an approach to automatically identify the morphological features of leave diseases in category of healthy to non-healthy based on region of interest of discolouration on young oil palm leaves. Raw leaf images are captured using a built-in digital camera. Pre-processing was done on each of the non-uniform illumination condition raw data images. We tested the colour feature method using RGB (Red, Green Blue) colour filtering in the identification of the leaf region of interest. Next, further segmentation method using HSV (Hue, Saturation, Values) colour filtering approach is employed to remove shadows and to identify the different level of regions of discolouration. The results highlighted that the infected area on the leaves can be identified by $100 \%$ based on the discoloured in the region of interest. These regions can be categorised in three different groups - healthy leaves $(20 \%$ of the discolouration region) to heavily infected $(70 \%$ of the discolouration region) of the leaves - based on analysis of the pre-processing results. In top of that, the HSV colour feature method could also remove shadow and noise. The results of the detected discolouration will be used oil palm leaves datasets for further classification and recognition research work.
\end{abstract}

Keywords - colour image segmentation, colour thresholding, RGB, HSV, oil palm leaves disease

\section{INTRODUCTION}

Agriculture is a consideration vital to the economy of Malaysia. This sector is the backbone of the Malaysian economy through the production of agricultural products for domestic consumption. According to statistics in 2019, agriculture contributes $7.1 \%$ (RM101.5 billion) to the national Gross Domestic Products (GDP). Of this total GDP, oil palm contributed $37.7 \%$ [1]. This statistic indicates that oil palm is the major contributor to the agricultural sector in Malaysia. However, agriculture is very susceptible to factors, such as pest infestation, disease, and climate change [2]. In a large-scale agriculture such as oil palm plantations, disease outbreaks will be a significant problem that may cause serious effects if the disease is not detected in an early stage. The quality and amount of oil palm seedlings can also be devastatingly affected. The diseased symptoms are visibly detectable through the change of colour, shape, or its function. The varying disease spot colours is very different from plant leaf colour, this it can be categorised from a health leaf to a heavily infected or dying leaf. It is therefore vital to detect diseases on the leaves at the early stage. In the research, work will focus on oil palm leaves disease detection in the nursery area. The oil palm is aged between two months until 12 months old.

Currently, the identification of plant disease based on the different categories of region of interest of the diseases is manually and visually identified by experts in the nursery. This observation commonly done by experts may not be the best approach as it is meticulously tedious and may lead to less accurate detection of the different level of diseases on the leaves. It requires a skilled and experience experts to identify the different categories of disease spots based on the visible morphology of the leaves. Here, we are proposing to replace the human visual approach to using image processing methods to detect this morphology of discolouration on leaves which then could be used further recognition and analysis. The existing methods to detect plant diseases in image pre-processing commonly use colour detection on the region of the infected areas [3]. To detect the infected areas, an image segmentation approach is used. It is a process of subdividing the whole image into different regions based on the wanted features, such as colour information, boundaries, or segment of an image [3]. There are various techniques and algorithms for the segmentation and detection of plant leaf disease [4], [5], [6]. The commonly used segmentation approach is the grey thresholding method. This algorithm works by selecting the pixels in the range as belonging to the foreground and rejecting all other pixels outside the region of interest in the background [7]. The final segmented representation is usually in a binary image, whereby black pixels correspond to the background, and white pixels correspond to the foreground/region of interests. The human eyes can differentiate thousands of colours but can only distinguish 20 shades of greyscale. Therefore, full Red, Green and Blue (RGB) colour model will be used to ensure details of information will not be accidentally removed in greyscale.

The work in this paper aims to analyse the use of RGB and HSV (Hue, Saturation, Value colour model) thresholding methods in image segmentation techniques on leaves to detect disease spots on the leaves. Filter values in image enhancement method are adjusted to reduce noise. Segmentation from the colour thresholding methods is employed to extract the region of interest, that is the disease spots, and shadows. Colours filters is used to enhance the disease spot colour. The paper is organised in four sections. Section II define and describe the proposed methods that consists of five main processes. The experimental results are showed and discussed, along with the analysed colour 